\title{
DIAGNÓSTICO AMBIENTAL DE LA PERCEPCIÓN DE LA CONTAMINACION VISUAL POR PARTE DE LA POBLACIÓN UNIVERSITARIA DE LA FACULTAD DE INGENIERÍA CIVIL Y DE LA FACULTAD DE CIENCIAS NATURALES, EXACTAS Y DE LA EDUCACIÓN DE LA UNIVERSIDAD DEL CAUCA
}

\author{
David Alejandro Mera Benavides ${ }^{1}$
}

Recibido el 12 de febrero de 2015, aprobado el 26 de abril de 2016 y actualizado el 20 de diciembre de 2016

DOI: 10.17151/luaz.2017.44.13

\section{RESUMEN}

En dos facultades de la Universidad del Cauca, fue realizado un diagnóstico ambiental sobre la percepción y el grado de contaminación visual que se tienen dentro de ellas, para lo anterior se utilizaron encuestas de opinión universitaria y pública para el conocimiento de diversos factores que generan, alteran y ofrecen soluciones al ambiente con la mencionada problemática. Los resultados porcentuales con la ayuda del programa estadístico SPSS demostraron como en su mayoría la población universitaria conoce sobre la importancia del impacto generado con un $84 \%$ y un $87 \%$ de las molestias, además que el $36,4 \%$ de la población encuestada establece que la educación es la solución para el problema. El estudio también establece que son los estudiantes universitarios los que están dispuestos para buscar soluciones y la mitigación de la contaminación visual a través de nuevos espacios.

Palabras clave: Contaminación visual, diagnóstico ambiental, mitigación, Universidad del Cauca, estudiantes universitarios.

\section{ENVIRONMENTAL DIAGNOSIS OF VISUAL POLLUTION PERCEPTION BY UNIVERSITY POPULATION FROM THE CIVIL ENGINEERING AND NATURAL, EXACT AND EDUCATION SCIENCES FACULTIES AT UNIVERSIDAD DEL CAUCA}

\section{ABSTRACT}

An environmental assessment of the perception and the degree of visual pollution in the campus, was conducted in two faculties of Universidad del Cauca. For this purpose, university and public opinion surveys to know different factors that generate, alter and offer solutions to the environment with the aforementioned problems were conducted. The percentage results using the SPSS statistical program showed how most of the university population knows about the importance of the impact generated with an $84 \%$ and an $87 \%$ of the discomfort, plus $36.4 \%$ of the population surveyed states that education is the solution to the problem. The study also establishes that it is the university students who are willing to seek solutions and mitigation of visual pollution through new spaces.

Key words: Visual pollution, environmental diagnosis, mitigation, Universidad del Cauca, university students. 


\section{INTRODUCCIÓN}

Colombia es un país de grandes oportunidades, en el que todos sus habitantes se pueden desenvolver con libre expresión, debido a que la democracia que rige en el territorio colombiano, es un sistema con el cual las personas están dispuestas a tener una "libertad" y "equidad" de sus acciones, pero al mismo tiempo es un lugar donde la desigualdad de las clases sociales se hace evidente, es por ello que en muchos aspectos la problemática ambiental es aún más grave, donde la responsabilidad sobre la contaminación es muy variable o desigual. Aquí se llega a que la democracia hace que todos "tengan los mismos derechos", donde la libre expresión, la libertad de opinión y protesta, la libertad de comunicación y publicidad se da sin ningún problema, pero es por otro contexto donde los efectos de todas estas acciones sociales y necesarias, generan un tipo de contaminación, en particular, la contaminación visual, sin ninguna concientización de lo que se está produciendo hacia el ambiente de manera perjudicial (Pereira, 2013). Este tipo de interacciones como son social, política, económica y cultural, conllevan a que las personas adquieren un tipo de pensamientos o necesidades que deben ser expresadas de acuerdo con el contexto en el que se desenvuelve cada individuo. Así, una de las formas usadas por los estudiantes, trabajadores y particulares para comunicar a los demás, están reflejadas en los grafitis, panfletos, carteleras, murales, afiches, vallas, etc. (Boff, 2013).

Colombia es uno de los países más diversos del mundo, en recursos naturales, como en sus lenguas nativas, en su vocabulario, en sus razas, en su cultura, entre muchos aspectos, por eso hoy en día muchos centros educativos, especialmente las universidades, son testigos de la diversidad de Colombia; debido a aquello, en cada salón, en cada asiento hay una pequeña cabeza que se está expresando a cada segundo de muchas maneras y estas dependen, de donde venga aquella persona, de su educación, de sus padres y amigos, de sus costumbres y creencias (Pereira, 2013). Popayán, Cauca, Colombia es una de las ciudades donde la diversidad cultural es un grado mediano notoria, podemos encontrar cualquier clase de expresión, en cualquier época del año, por cualquier eventualidad, tal vez por las situaciones difíciles que ha pasado la ciudad en sus últimos años, como el desempleo y la violencia, además de las costumbres tradicionales que siempre han sido objeto de estudio, desde un evento musical hasta un acto religioso, son aquellas situaciones en donde la comunicación se hace presente de diversas maneras, pues donde haya relaciones culturales, habrá comunicación, al igual que en las relaciones políticas y económicas, por muy buenas o desagradables que sean, por eso tal vez es en la "Ciudad blanca de Colombia" donde la expresión cuenta, una ciudad que ha quedado un poco olvidada, a pesar de su historia, que cuenta con la sexta mejor universidad del país, pero donde el Estado ha quedado corto para su mejoramiento o mantenimiento; es desde estas situaciones donde la génesis de la contaminación visual se hace aún más notoria y negativa.

La contaminación visual es todo aquello que afecta la visualización de un determinado lugar, o vaya en contra de la estética de una zona o paisaje, causando un problema que incluso puede afectar la salud de quienes conviven en este lugar. Esta contaminación tiene como referencia a los carteles y vallas publicitarias, que dependiendo de su tamaño y exceso se convierten en un problema. La preocupación por la contaminación visual que afecta áreas urbanas, es decir, al ambiente construido que da cabida a la mayor parte de la población en Latinoamérica $(79,43 \%)$ y el mundo $(50,46 \%)$, acorde con los datos de Naciones Unidas (2009) (Peña, 2010).

El consumismo, que siempre está presente en la sociedad, conlleva a este tipo de acciones, así mismo la expresión de ideas o pensamientos de personas que sin conciencia social actúan sin pensar en si sus acciones están bien hechas o no, 
mediante los grafitis con contenidos inapropiados muchas veces y que a la vez conllevan a las infraestructuras hacia un deterioro (Bookchin, 1974; Tzonis 1977).

La saturación publicitaria es el principal agente de distorsión y de contaminación visual y ambiental en el ecosistema urbano. El exceso publicitario no es sólo un elemento contaminante del campo visual; hoy día la publicidad en la urbe perturba todos los sentidos, por lo que hablar únicamente de contaminación visual puede no reflejar la verdadera dimensión del problema (Olivares, 2009). Son así las carteleras publicitarias las que generan un impacto más inmediato en el consumidor; esta es la razón por la cual se observa en mayores cantidades sobre las paredes o fachadas de las infraestructuras, destruidas u ocultas por la superposición de carteles (Arbohaín y Garcén, 2001; Díaz, 1997).

Además de la cuestión estética y del deterioro paisajístico y ecológico, algunos estudios empíricos empiezan a señalar que el exceso de publicidad está afectando la salud de los ciudadanos. En el caso de los animales, diversas asociaciones ecologistas mantienen que el exceso de anuncios y de colorido estridentes, en lugares de paso de la fauna, pueden alejar algunas especies e incluso romper el equilibrio ecológico (Olivares, 2009).

Esta investigación plantea la identificación de impactos negativos generados por contaminación visual dentro de las facultades de Ingeniería Civil y la de Ciencias Naturales, Exactas y de la Educación pertenecientes a la Universidad del Cauca en un contexto estudiantil, demarcado por diversos problemas, que son producto de una interacción social, política, económica y cultural.

\section{OBJETIVOS}

\section{Objetivo general:}

Diagnosticar la percepción de la contaminación visual dentro del contexto de dos facultades de la Universidad del Cauca.

\section{Objetivos específicos:}

- Identificar las posibles problemáticas visuales que se están generando en el bienestar universitario por parte del exceso de información visual.

- Indagar con la población universitaria sobre la importancia de las afectaciones de la contaminación visual y el conocimiento que se tiene sobre la misma.

- Determinar la disponibilidad a pagar (DAP) y el apoyo a la mitigación por parte de la población universitaria ante los impactos generados por la contaminación visual. 


\section{MATERIALES Y MÉTODOS}

Dentro del contexto universitario se tomó la contaminación visual que se presenta a diario por parte de los estudiantes, tal como son los avisos publicitarios de eventos, objetos perdidos, ventas, información de interés social, cultural y deportivo, en lugares donde no es permitido o no tienen la función de ofrecer este tipo de servicio, además de dibujos y grafitis que se realizan como voces de protestas de las necesidades de los mismos estudiantes, de mostrar mofas y burlas a figuras públicas nacionales y locales o por el mismo hecho del vandalismo interno dentro de la institución que está degenerando el patrimonio de la misma universidad; todo lo anterior se puede evidenciar en las siguientes imágenes:

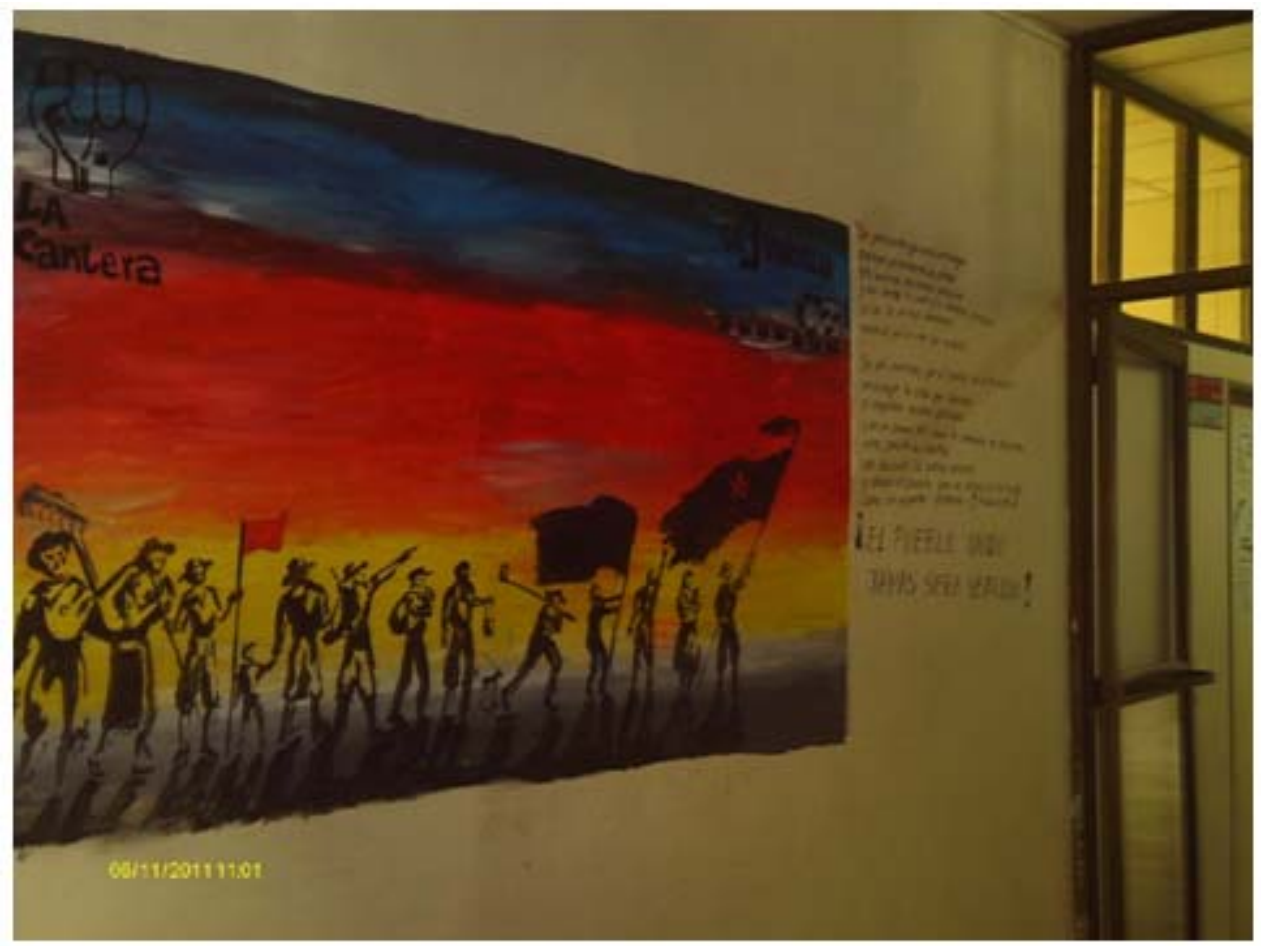

Fuente: esta investigación.

Figura 1. Dibujo de movimiento estudiantil en la Facultad de Ingeniería Civil. 


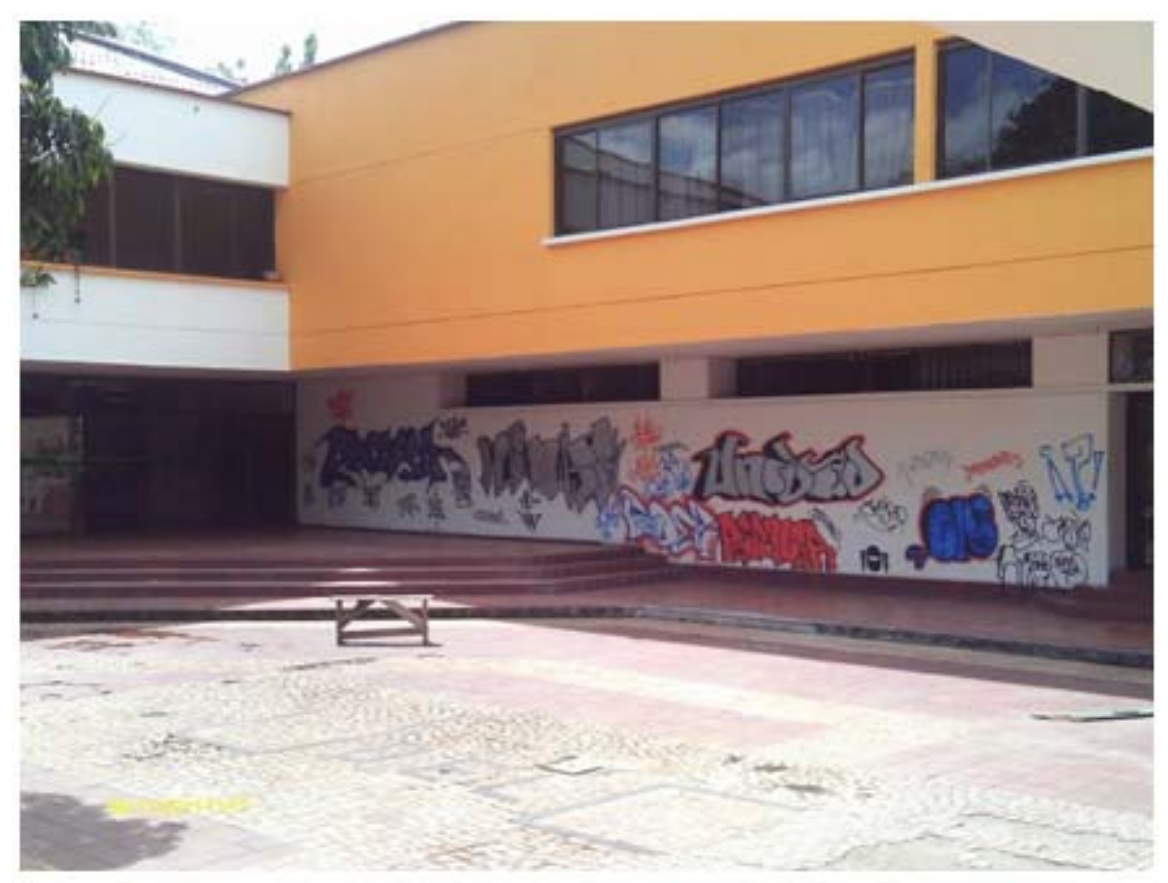

Fuente: esta investigación.

Figura 2. Grafitis en la Facultad de Ciencias

Naturales, Exactas y de la Educación.

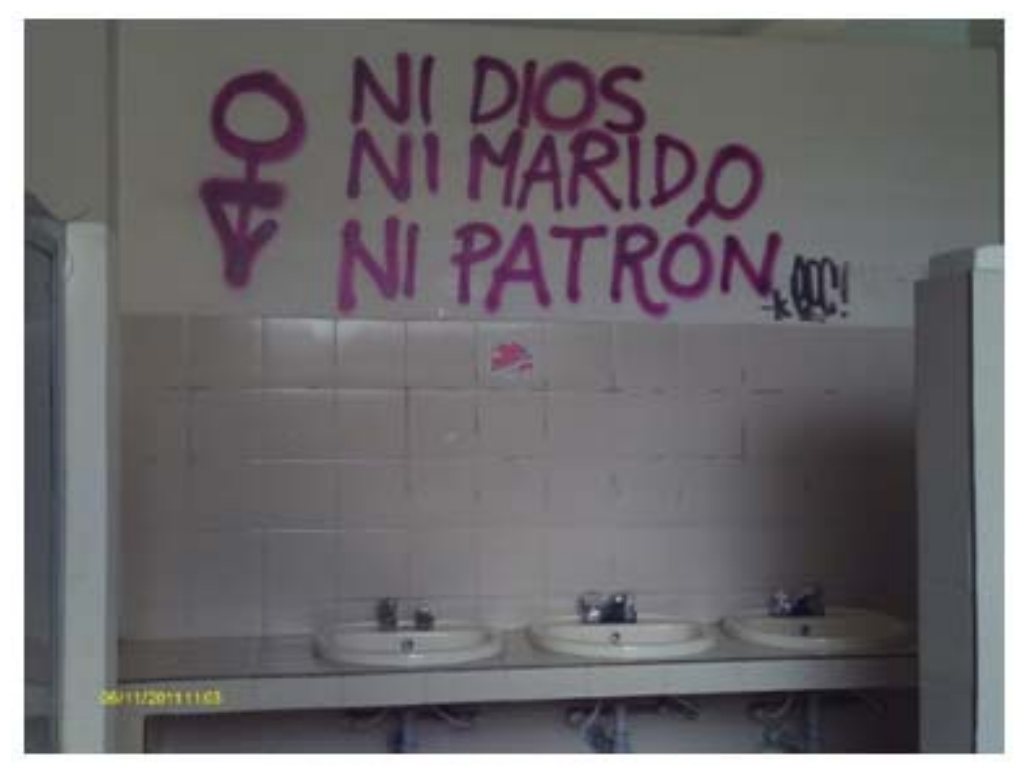

Fuente: esta investigación.

Figura 3. Grafitis en baño de la Facultad de Ciencias Naturales, Exactas y de la Educación. 


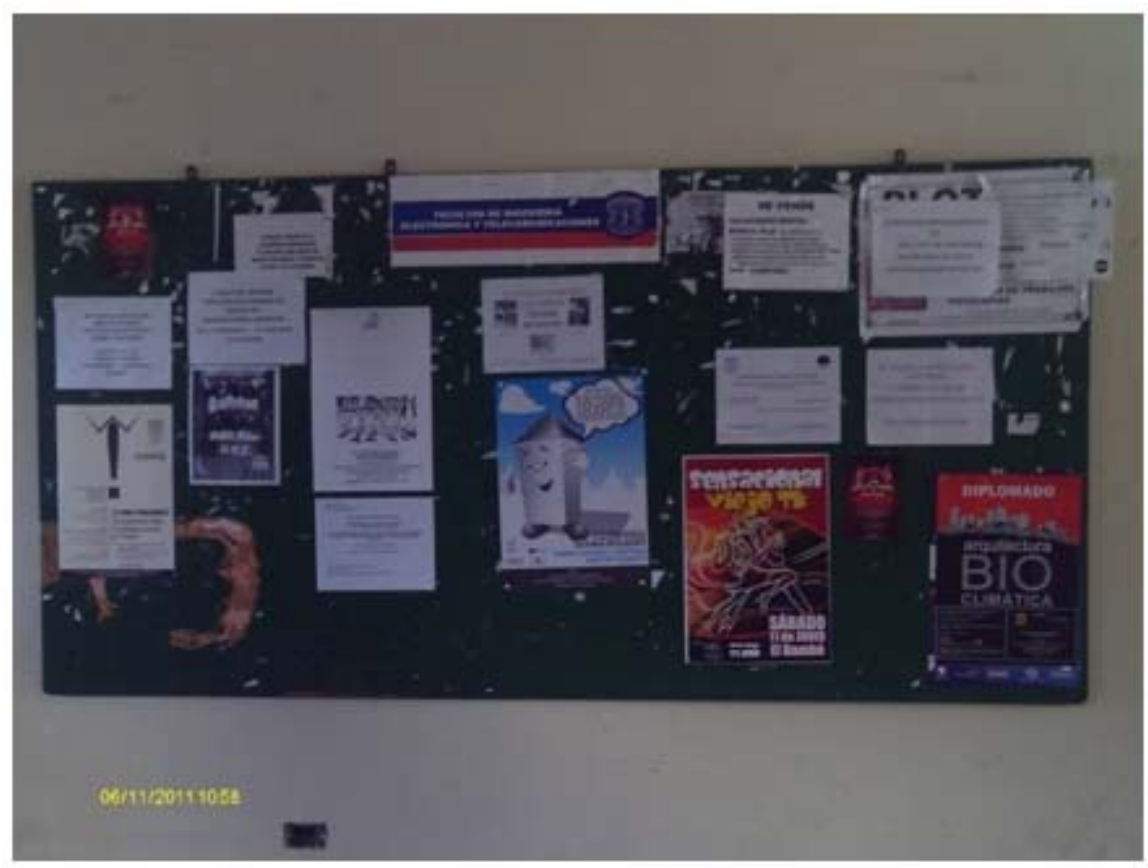

Fuente: esta investigación.

Figura 4. Publicidad en la Facultad de Ingeniería Civil.

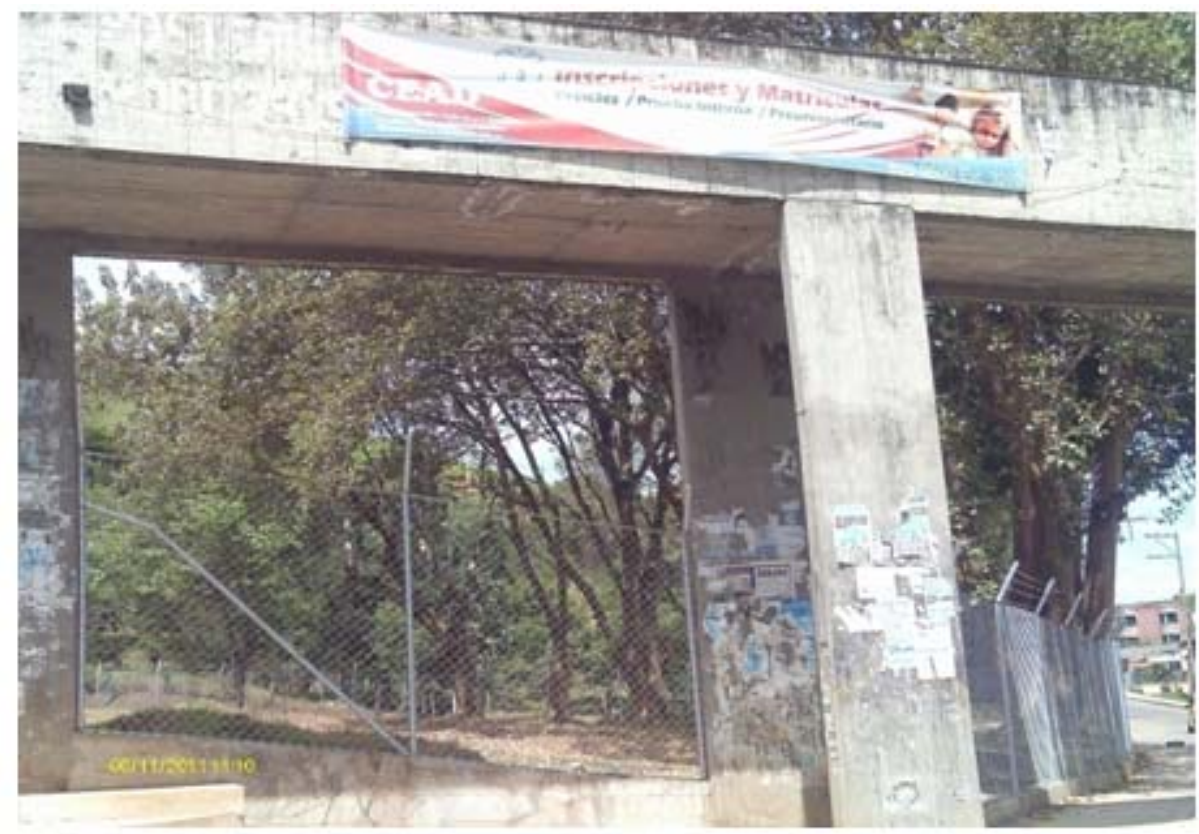

Fuente: esta investigación.

Figura 5. Publicidad en el puente que une a las dos facultades.

Para el presente estudio realizado en la Universidad del Cauca solo se tuvieron en cuenta dos de sus nueve facultades, debido a que la población de estudiantes en las dos facultades escogidas, Ingeniería Civil y Ciencias Naturales, Exactas y de la Educación, son las que poseen la mayor población de estudiantes. Es en ellas donde por la diversidad de la población, los indicadores socioeconómicos son los que más se 
ven reflejados en las carteleras y grafitis, los cuales se realizan con el objetivo de brindar productos y servicios además de expresar inconformidad ante las políticas nacionales en forma de protestas y quejas antes las mismas.

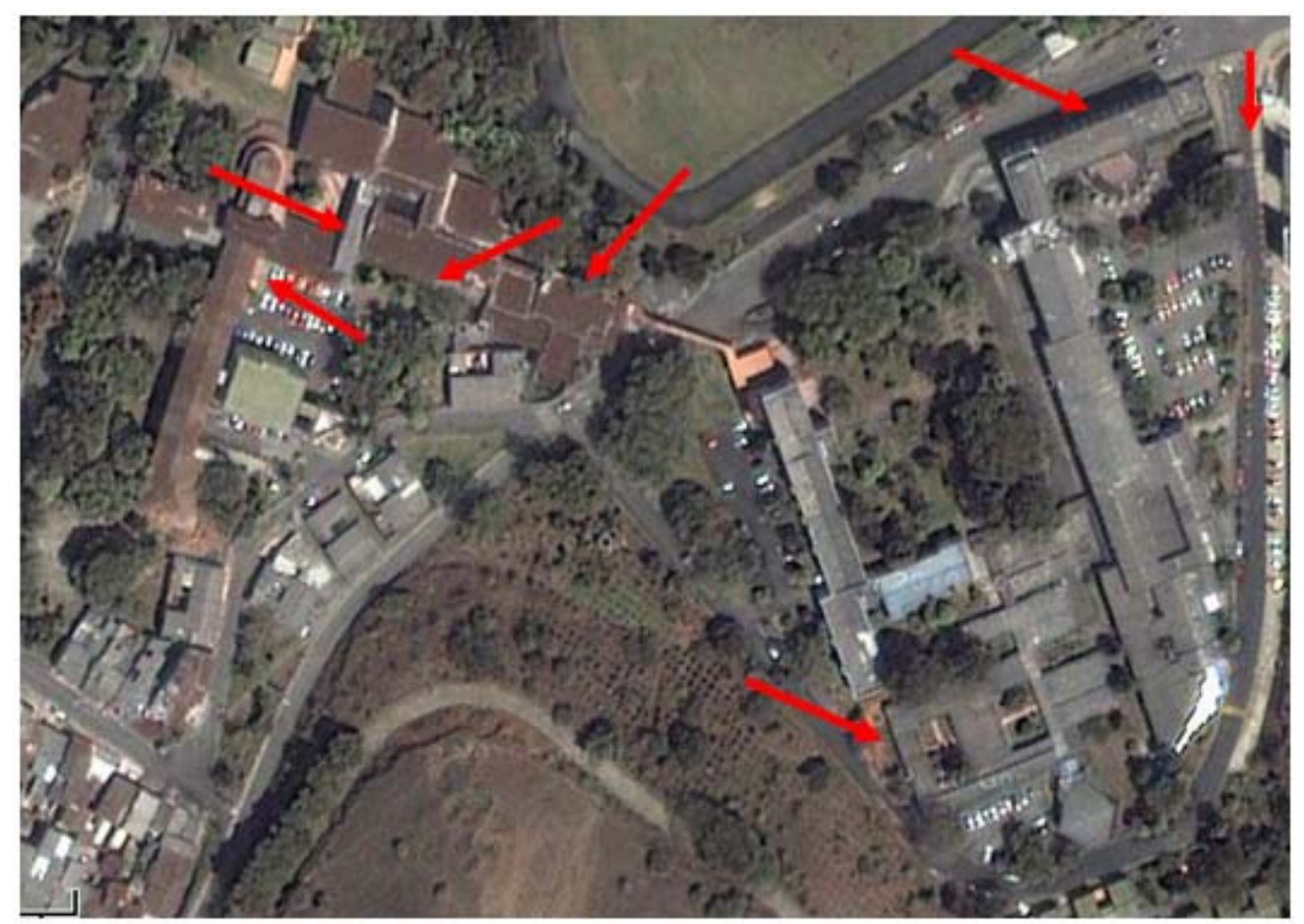

Fuente: (Google Earth, 2013)

Figura 6. Mapa de la Facultad de Ingeniería Civil y la Facultad de Ciencias.

Naturales, Exactas y de la Educación

En esta investigación sobre el diagnóstico ambiental de la contaminación visual en las facultades de Ingeniería Civil y Ciencias Naturales, Exactas y de la Educación, las herramientas para percibir el impacto ambiental que se está generando en estos dos sectores de la Universidad del Cauca fueron las encuestas realizadas a los actores que están permanentemente en el campus, tal como son estudiantes y docentes de los diferentes programas de pregrado y posgrado. Para ello se realizaron un total de 67 encuestas, para la evaluación independiente de los estudiantes (33 encuestados) y docentes (34 encuestados), de esta forma se está al tanto del grado de conocimiento sobre el concepto y definición de contaminación visual, además de la concientización sobre los impactos ambientales que se están generando dentro del campus universitario y la DAP para la mitigación y control de esta problemática ambiental; además se encuestaron a 33 personas particulares, evaluándoles los mismos parámetros anteriormente nombrados y así conocer el grado de concientización de las personas que no pertenecen a la institución directamente.

A continuación se presentan la información de la población y la densidad poblacional calculada de estudiantes y docentes de la Universidad del Cauca en el historial de los últimos semestres: 
Tabla 1. Estudiantes de pregrado matriculados por facultad en el año 2013.

\begin{tabular}{|c|c|c|}
\hline FACULTAD & 12013 & 12013 \\
\hline ARTES & 668 & 700 \\
\hline CIENCUS AGROPECUARIAS & 1166 & 1120 \\
\hline CIENCAS CONTABLES ECONOMCAS Y ADMNNISTRATNAS & 1500 & 1540 \\
\hline Cievcuas de La saluo & 1705 & 1609 \\
\hline CIENCLAS HUMANAS Y SOCHALS & 1494 & 1490 \\
\hline CENCIAS NATURALES, DUCTAS Y DE LA EDUCACION & 1885 & 1949 \\
\hline DERECHO Y CIENCLAS POUTCAS Y SOCIALES & 2114 & 20303 \\
\hline NGENIERIA CMLL & 1286 & 1286 \\
\hline INGENERAA ELECTRONCA Y TELECONUNACACIONES & 1560 & 1545 \\
\hline TOTAL & 13.378 & 13.652 \\
\hline
\end{tabular}

Fuente: (Universidad del Cauca, 2013)

Tabla 2. Docentes y tipo de vinculación por facultad en el año 2013.

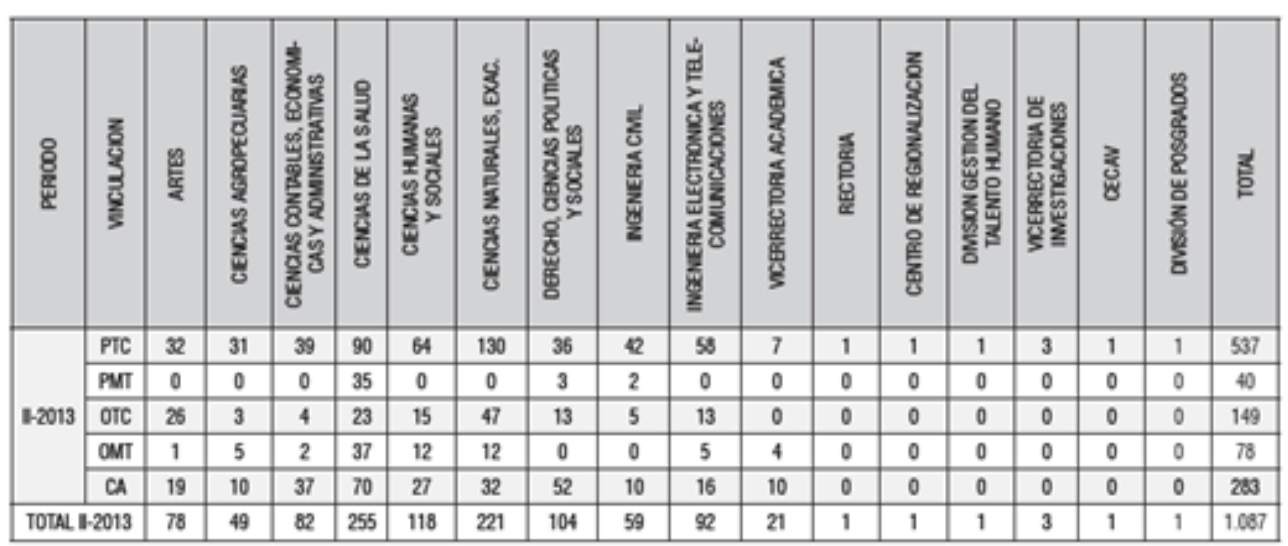

Fuente: (Universidad del Cauca, 2013)

- Aproximación del área superficial de la Facultad de Ingeniería Civil: 10035 m².

- Aproximación del área superficial de la Facultad de Ciencias Naturales, Exactas y de la Educación: $17978 \mathrm{~m}^{2}$.

- Aproximación de los habitantes de la Facultad de Ingeniería Civil: 1500 habitantes.

- Aproximación de los habitantes de la Facultad de Ciencias Naturales, Exactas y de la Educación: 2300 habitantes.

- Densidad poblacional de la Facultad de Ingeniería Civil: 6,7 habitantes $/ \mathrm{m}^{2}$.

- Densidad poblacional de la Facultad de Ciencias Naturales, Exactas y de la Educación: 7,8 habitantes $/ \mathrm{m}^{2}$.

(Universidad del Cauca, 2013). 
Para realizar el análisis cuantitativo y cualitativo de los resultados y de todas las interrelaciones, gráficos y porcentajes se utilizó el software estadístico Statistical Package for the Social Sciences (SPSS).

\section{RESULTADOS Y DISCUSIÓN}

Los siguientes resultados con su respectiva discusión hacen referencia a cada una de las preguntas presentadas en las 100 encuestas realizadas, aunque las tablas 3 y 4 y las gráficas hacen referencia a lo obtenido en relación con el sexo y ocupación de las personas que se encuestaron.

Tabla 3.Resultadosde las personas encuestadas con respecto a su sexo o género.

\section{SEXO}

\begin{tabular}{|r|r|r|r|r|r|}
\hline \multicolumn{2}{|c|}{} & Frecuencia & Porcentaje & $\begin{array}{r}\text { Porcentaje } \\
\text { válido }\end{array}$ & $\begin{array}{r}\text { Porcentaje } \\
\text { acumulado }\end{array}$ \\
\hline Válidos & Masculino & 59 & 59 & 59 & 59 \\
\cline { 2 - 6 } & Femenino & 41 & 41 & 41 & 100 \\
\cline { 2 - 6 } & Total & 100 & 100 & 100 & \\
\hline
\end{tabular}

\section{Fuente: elaboración propia}

\section{SEXO}

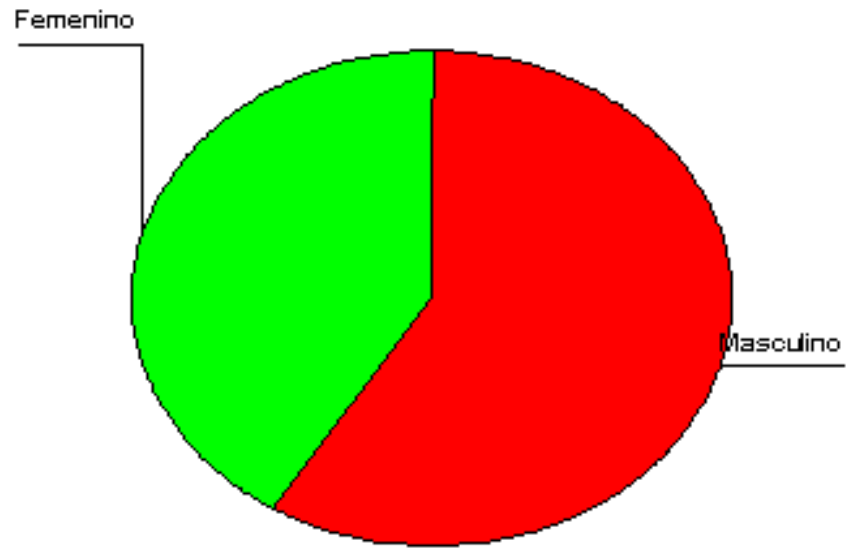

Fuente: elaboración propia

Figura 7. Diagrama de sectores de las personas encuestadas con respecto a su sexo o género. 
La tendencia a encuestar a las personas siempre se tuvo que fuera en un $50 \%$ para mujeres y un $50 \%$ para hombres, pero la disponibilidad del tiempo de las personas no siempre era la misma, en especial de la planta docente, por esta razón hay una diferencia de $18 \%$ entre los dos géneros de personas encuestados, es decir que hay una diferencia estadística significativa.

Tabla 4. Resultados de las personas encuestadas

con respecto a su ocupación.

\section{OCUPACIÓN}

\begin{tabular}{|l|l|r|r|r|r|}
\hline \multicolumn{2}{|c|}{} & Frecuencia & Porcentaje & \multicolumn{1}{c|}{$\begin{array}{c}\text { Porcentaje } \\
\text { válido }\end{array}$} & $\begin{array}{c}\text { Porcentaje } \\
\text { acumulado }\end{array}$ \\
\hline Válidos & Estudiante & 33 & 33 & 33 & 33 \\
\cline { 2 - 6 } & Docente & 34 & 34 & 34 & 67 \\
\cline { 2 - 6 } & Particular & 33 & 33 & 33 & 100 \\
\cline { 2 - 6 } & Total & 100 & 100 & 100 & \\
\hline
\end{tabular}

\section{Fuente: elaboración propia}

\section{OCUPACIÓN}

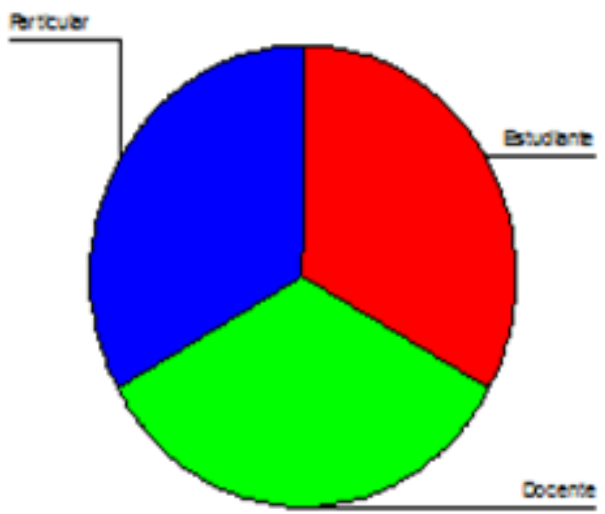

Fuente: elaboración propia

Figura 8. Diagrama de sectores de las personas encuestadas

\section{con respecto a su ocupación}

En la metodología de la investigación se definió que la muestra poblacional fuera de 100 personas encuestadas, por lo tanto para cada ocupación el número de encuestas es el mismo, a excepción de los docentes que tuvieron una encuesta más que los estudiantes y particulares, es decir un $1 \%$ de más, diferencia que no es significativa para el análisis de las preguntas. 
Tabla 5. Resultados de porcentaje de contingencia de las personas encuestadas con respecto a la ocupación y al conocimiento sobre el concepto y la definición de la contaminación visual.

\section{¿SABE QUÉ ES? vs OCUPACIÓN}

Recuento

\begin{tabular}{|l|l|r|r|r|r|}
\hline \multicolumn{2}{|c|}{} & \multicolumn{3}{|c|}{ OCUPACION } & \multirow{2}{*}{ Total } \\
\cline { 3 - 5 } \multicolumn{2}{|c|}{} & Estudiante & Docente & Particular & \multicolumn{1}{c|}{ 'SABE } \\
QUEES? & No & 4 & 5 & 7 & 16 \\
\cline { 2 - 5 } & SI & 29 & 29 & 26 & 84 \\
\hline Total & 33 & 34 & 33 & 100 \\
\hline
\end{tabular}

Fuente: elaboración propia

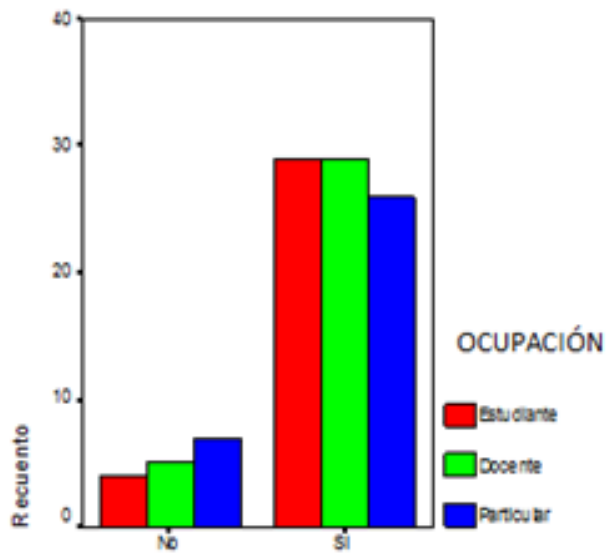

¿SABE QUÉ ES?

Fuente: elaboración propia

Figura 9. Diagrama de barras de contingencia de las personas encuestadas con respecto a la ocupación y al conocimiento sobre el concepto y la definición de la contaminación visual.

Con respecto a la primera pregunta de cada una de las encuestas que decía: ¿Sabe qué es contaminación visual?, se muestra un $84 \%$ de conocimiento de todos los encuestados que respondieron positivamente a la pregunta, tan solo 16 personas no sabían qué era la contaminación visual, pero se muestra que los estudiantes y los docentes son las personas que más tienen conocimiento sobre esta problemática, mientras que personas ajenas a la Universidad del Cauca, tenían un menor conocimiento. Se puede decir que la contaminación visual es uno de los problemas que se conoce dentro de la universidad, mas no se puede asegurar que las personas sepan qué y quiénes están provocando dicha contaminación. 
Tabla 6. Resultados de contingencia de las personas encuestadas con respecto al sexo o género y a la generación de molestias por la contaminación visual.

\section{¿LE GENERA MOLESTIAS? VS SEXO}

\begin{tabular}{|c|c|c|c|c|}
\hline & & \multicolumn{2}{|c|}{ SEXO } & \multirow[b]{2}{*}{ Total } \\
\hline & & Masculino & Femenino & \\
\hline \multirow{2}{*}{$\begin{array}{l}\text { ¿LEGENERA } \\
\text { MOLESTIAS? }\end{array}$} & No & 6 & 7 & 13 \\
\hline & Sí & 53 & 34 & 87 \\
\hline \multicolumn{2}{|l|}{ Total } & 59 & 41 & 100 \\
\hline
\end{tabular}

Fuente: elaboración propia

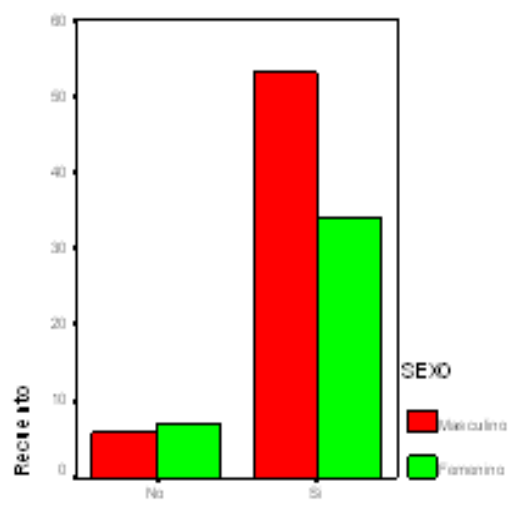

¿LE GENERA MOLESTIAS?

Fuente: elaboración propia

Figura 10. Diagrama de barras de contingencia de las personas encuestadas con respecto al sexo o género y a la generación de molestias por la contaminación

visual.

Los impactos negativos a las personas de la contaminación visual se hacen evidentes; el estrés y mal aspecto fueron dos de los muchos conceptos que se respondieron en las encuestas. En esta gráfica se muestra que tanto para mujeres como para hombres, hay generación de molestias provocadas por la contaminación visual. 
Tabla 7. Resultados de contingencia de las personas encuestadas con respecto a la ocupación y a la generación de molestias por la contaminación visual.

\section{¿LE GENERA MOLESTIAS? vs OCUPACIÓN}

\begin{tabular}{|c|c|c|c|c|c|}
\hline & & \multicolumn{3}{|c|}{ OCUPACION } & \multirow[b]{2}{*}{ Total } \\
\hline & & Estudiante & Docente & Particular & \\
\hline \multirow{2}{*}{$\begin{array}{l}\text { ¿LE } \\
\text { GENERA } \\
\text { MOLESTIAS } \\
?\end{array}$} & No & 3 & 4 & 6 & 13 \\
\hline & Sí & 30 & 30 & 27 & 87 \\
\hline Total & & 33 & 34 & 33 & 100 \\
\hline
\end{tabular}

Fuente: elaboración propia

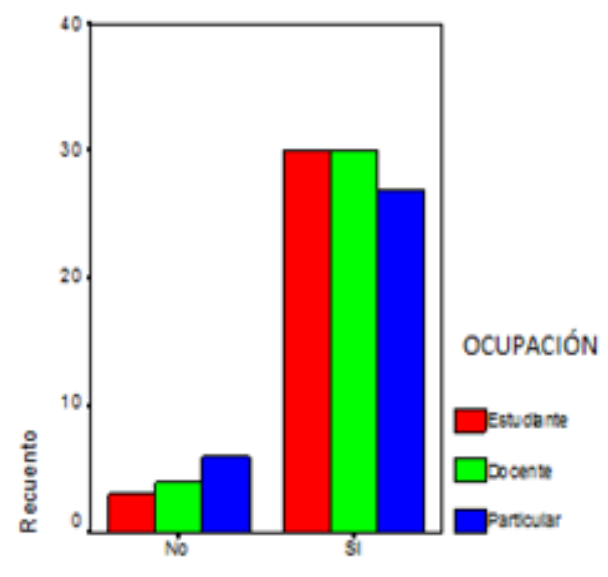

¿LE GENERA MOLESTAS?

Fuente: elaboración propia

Figura 11. Diagrama de barras de contingencia de las personas encuestadas con respecto a la ocupación y a la generación de molestias por la contaminación visual.

Estos resultados son más específicos, con respecto a la pregunta sobre si la contaminación visual está provocando en las 100 personas encuestadas molestias o problemas, aquí se puede apreciar como la contaminación visual generada en las facultades indagadas, afecta especialmente a docentes y estudiantes en un porcentaje del $30 \%$ para cada uno, mientras que a las personas externas a la universidad, se observa en menos proporción la provocación de molestias; por esta razón, la no apreciación de los impactos ambientales generados por la contaminación visual, se mira en las personas particulares, mientras que los estudiantes son los mayores afectados. 
Tabla 8. Resultados de los estudiantes encuestados con respecto a la opinión sobre quiénes son las personas más perjudicadas por la contaminación visual.

\section{¿A QUIÉNES LES AFECTARIA MÁS?}

\begin{tabular}{|l|l|r|r|r|r|}
\hline \multicolumn{2}{|c|}{} & Frecuencia & Porcentaje & $\begin{array}{c}\text { Porcentaje } \\
\text { válido }\end{array}$ & $\begin{array}{r}\text { Porcentaje } \\
\text { acumulado }\end{array}$ \\
\hline Válidos & Estudiantes & 16 & 48,5 & 48,5 & 48,5 \\
\cline { 2 - 6 } & Particulares & 4 & 12,1 & 12,1 & 60,6 \\
\cline { 2 - 6 } & Aseadores & 1 & 3,0 & 3,0 & 63,6 \\
\cline { 2 - 6 } & Planta & 12 & 36,4 & 36,4 & 100 \\
& Física & 33 & 100 & 100 & \\
\cline { 2 - 6 } & Total & 33 & & & \\
\hline
\end{tabular}

\section{Fuente: elaboración propia}

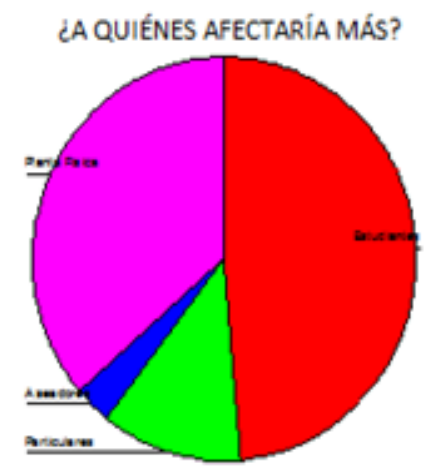

Fuente: elaboración propia

Figura 12. Diagrama de sectores de los estudiantes encuestados con respecto a la opinión sobre quiénes son las personas más perjudicadas por la contaminación visual.

Para los estudiantes, los más afectados por la contaminación visual son ellos mismos, debido a que el mal aspecto de la misma universidad genera un desequilibrio social y benéfico en la relación universidad - estudiante, además del mal manejo de la distribución de la información y del impedimento que se genera puesto que unos tienen más espacio publicitario que otros, por ejemplo en las carteleras. Además, la mala presentación de los salones y la falta de ética que se observa en los mismos; la planta física es el segundo factor al cual le afecta más la contaminación visual según la opinión de los estudiantes, esto se debe a que por el mal manejo de la información en la infraestructura de las facultades se ve afectado el repello de las paredes, además de suciedad de las mismas, incremento en la renovación de pintura de las mismas, además de los residuos que se generan de esta contaminación (aunque muchos de ellos son reciclables). Es decir, que tanto los estudiantes como la planta física son los 
más afectados y ambos son los miembros y las instalaciones respectivamente de las facultades estudiadas.

Tabla 9. Resultados de los estudiantes encuestados con respecto a la opinión sobre las posibles soluciones para la mitigación de la contaminación visual.

\section{¿CUÁL CREE QUE ES UNA POSIBLE SOLUCIÓN?}

\begin{tabular}{|l|l|r|r|r|r|}
\hline \multicolumn{2}{|c|}{} & Frecuencia & Porcentaje & $\begin{array}{c}\text { Porcentaje } \\
\text { válido }\end{array}$ & $\begin{array}{c}\text { Porcentaje } \\
\text { acumulado }\end{array}$ \\
\hline Válidos & $\begin{array}{l}\text { Espacios Libre } \\
\text { Expresión }\end{array}$ & 9 & 27,3 & 27,3 & 27,3 \\
\cline { 2 - 6 } & Educación & 12 & 36,4 & 36,4 & 63,6 \\
\cline { 2 - 6 } & Normatividad & 10 & 30,3 & 30,3 & 93,9 \\
\cline { 2 - 6 } & Seguridad & 2 & 6,1 & 6,1 & 100,0 \\
\cline { 2 - 6 } & Total & 33 & 100,0 & 100,0 & \\
\hline
\end{tabular}

Fuente: elaboración propia

\section{¿CUÁL CREE QUE ES LA SOLUCIÓN?}

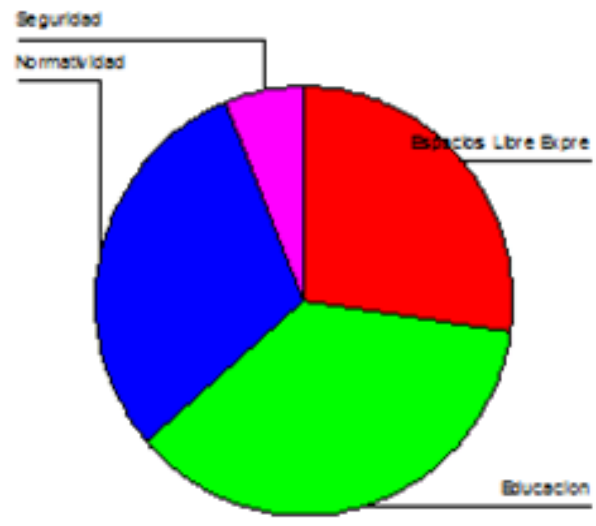

Figura 13. Diagrama de sectores de los estudiantes encuestados con respecto a la opinión sobre las posibles soluciones para la mitigación de la contaminación visual.

Los estudiantes son los que siempre buscan las soluciones cuando hay o existe algún problema de cualquier índole y para ellos en su mayoría, todo empieza por la educación, en su mayoría con un porcentaje del $36.4 \%$, dijeron que para empezar a mitigar el impacto de la contaminación visual en la universidad, debe realizarse una educación sobre dicha problemática, es decir, que antes que aportar dinero o prohibiciones, debe educarse, para así evitar los anteriores dos puntos, aunque tanto la reglamentación de normatividad como los espacios para la libre expresión de los estudiantes, tienen porcentajes cercanos al de la educación, por lo tanto en un buen 
plan de manejo ambiental, deben tenerse estos tres parámetros para que contribuyan a la mitigación de este problema estudiado, antes que implementar mayor seguridad en la universidad, la cual no es una salida viable a esta problemática.

Ver la concientización con respecto al problema de la contaminación visual dentro y fuera de la Universidad del Cauca, como solución integral no es del todo viable, ya que como es de esperarse, muchos responderán directa, indirectamente o desinteresadamente. Este último tipo de expresiones llevadas a la acción son las que más se observan en este mundo y por ello no se ven resultados del todo positivos, sino más bien grandes penas. Al partir de este hecho es importante entonces plantear normas por parte de interesados, junto con las directivas de la Universidad del Cauca, para lograr mitigar eficientemente el abuso de lo que muchos argumentan como libre expresión.

La idea no radica en impedir a los estudiantes que puedan expresar sus criterios y demás, sino más bien mitigar este tipo de excesos que se ven reflejados en las retaliaciones, rasguños y toda suerte de mensajes en los muros, pasillos, puertas, baños y demás; requerir la ayuda de vigilantes con el apoyo de dispositivos de seguridad tales como cámaras, entre otros objetos que hagan efectiva dicha labor, en la que se puedan verificar autores directos implicados en este tipo de acciones, de tal manera que se dé como sanción a dichas personas o bien a dichos programas académicos o facultades, la tarea de reparar lo que ha sido deteriorado por los mismos. De no hacerlo, implantar entonces un aumento en el pago de sus matrículas para el futuro semestre.

Otro de los aspectos a considerar sería la creación de un sitio web, a manera de red social que solo admita interactuar específicamente a estudiantes de la universidad, en la cual se puede exponer cualquier tipo de publicidad, o bien cualquier tipo de pensamiento que refleje inconformidad o conformidad. Es importante mencionar que este tipo de medio virtual permitiría intercambiar multitud de ideas por medio de los foros. Esta herramienta resultaría muy viable, primero que todo porque los estudiantes en especial utilizan con gran auge el internet, además de que quizá posibilitaría una significativa reducción en lo que alude a la contaminación visual dentro y fuera de la universidad en general.

La decisión por parte de los estudiantes es otro punto importante para mencionar ya que esto implica determinación por parte de los mismos, para enviar por medio de cartas o de cualquier otro medio permisible, a las autoridades municipales o gubernamentales, cualquier inconformidad que se haya derivado por decisiones de tipo político o similar. Este tipo de medidas contribuyen de alguna manera a mitigar la contaminación visual por exceso de publicidad, rayones, mensajes entre otros, dentro y fuera de nuestra universidad. El objetivo también considera el exponer nuestras inconformidades ante alguien y no siempre pelear u hostigar con el contenido de dichos mensajes a personajes, institutos $u$ organizaciones que con mayor probabilidad ni escucharán, y por ende tampoco responderán. 
Tabla 10. Resultados de porcentaje de las personas encuestadas con respecto a la disponibilidad a pagar (DAP).

¿COLABORARIA PARA LA MITIGACIÓN?

\begin{tabular}{|l|l|r|r|r|r|}
\hline \multicolumn{2}{|c|}{} & Frecuencia & Porcentaje & $\begin{array}{l}\text { Porcentaje } \\
\text { válido }\end{array}$ & $\begin{array}{r}\text { Porcentaje } \\
\text { acumulado }\end{array}$ \\
\hline \multirow{3}{*}{ Válidos } & No & 20 & 20 & 20 & 20 \\
\cline { 2 - 6 } & Si & 80 & 80 & 80 & 100 \\
\cline { 2 - 6 } & Total & 100 & 100 & 100 & \\
\hline
\end{tabular}

Fuente: Elaboración Propia

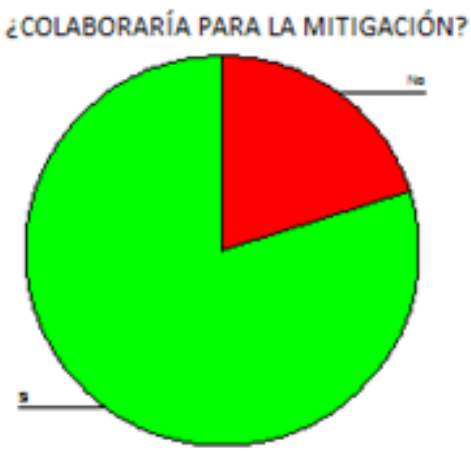

Fuente: elaboración propia

Figura 14. Diagrama de sectores porcentaje de personas encuestadas con respecto a la disponibilidad a pagar (DAP).

Con la DAP o la colaboración por la mitigación de la contaminación visual dentro de la Universidad del Cauca, existe un $80 \%$ de las 100 personas encuestadas, que están dispuestas a la colaboración por la mitigación de dicho problema, es decir, que la mayoría de las personas están en contra de esta problema, que quieren soluciones y que están dispuestas a ayudar de alguna forma (económica o laboral) para el desarrollo de diferentes estrategias ambientales para la regulación de esta acción; existe un $20 \%$ que no está dispuesta a pagar, lo se puede deber a que este problema no les está ocasionando dificultades. 
Tabla 11. Resultados de porcentaje de contingencia de personas encuestadas con respecto a la ocupación y a la DAP para la mitigación de la contaminación visual.

\section{¿COLABORARÍA PARA LA MITIGACIÓN? vS OCUPACIÓN}

Recuento

\begin{tabular}{|l|l|r|r|r|r|}
\hline \multicolumn{2}{|c|}{} & \multicolumn{3}{|c|}{ OCUPACION } & \multirow{2}{*}{ Total } \\
\cline { 3 - 6 } \multicolumn{2}{|c|}{} & Estudiante & Docente & Particular & \\
\hline \multirow{2}{*}{$\begin{array}{l}\text { ¿COLABORAR } \\
\text { IA PARA LA } \\
\text { MITIGACION? }\end{array}$} & No & 2 & 7 & 11 & 20 \\
\cline { 2 - 5 } & Si & 31 & 27 & 22 & 80 \\
\hline Total & 33 & 34 & 33 & 100 \\
\hline
\end{tabular}

Fuente: elaboración propia

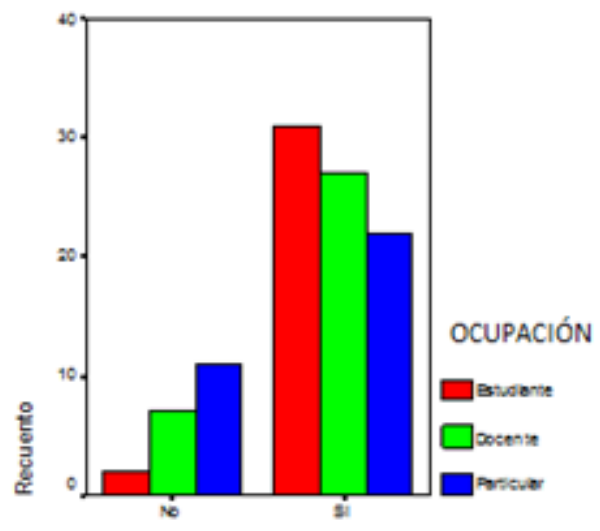

¿COLABORARÍA PARA LA MITIGACIÓN?

Fuente: elaboración propia

Figura 15. Diagrama de barras de contingencia del porcentaje de personas encuestadas con respecto a la ocupación y a la DAP para la mitigación de la contaminación visual.

Los estudiantes y los profesores están dispuestos a la colaboración en la mitigación de la contaminación visual, debido a que son los más afectados dentro de sus facultades, por esta razón su colaboración se hace mayoritaria en comparación que los particulares, quienes tienen menor participación en ellas; esto se puede determinar debido a que no pertenecen a la institución educativa, además de que muchos de ellos pueden no tener familiares dentro de la universidad, es decir que la acción de DAP se hace evidente en los afectados especialmente. 


\section{CONCLUSIONES Y RECOMENDACIONES}

La contaminación visual por el exceso de publicidad, grafitis y demás dentro y fuera de la Universidad del Cauca, atendiendo fundamentalmente a la Facultad de Ingeniería Civil y la Facultad de Ciencias Naturales y Exactas de la Educación, no está limitada en ninguna norma por parte de la misma institución; es precisamente esta situación la que se habría de considerar para hablar de un manejo ambiental con respecto a dicho problema, ya que la contaminación visual es un problema que como se ha descrito en este trabajo afecta desde diferentes puntos de vista a la misma comunidad universitaria e incluso también a particulares.

La población universitaria es quien tiene la concepción y percepción ideal de la contaminación visual dentro de la misma Universidad del Cauca y que demuestra que no están lejos ni tampoco desinformadas y que están afectadas sobre esta problemática que se vive cada día en la institución, lo cual ha generado también las quejas del vandalismo que se vive dentro de la misma institución con el que destruyen y afectan los bienes físicos de la universidad.

La DAP es fuertemente positiva en la muestra de la población encuestada pues le interesa aportar para contrarrestar los daños físicos, visuales y saludables de la universidad como de quienes hacen parte de ella por causa de este tipo de contaminación, pero este trabajo no alcanzó a cubrir el estudio del proceso administrativo y financiero al obtener dichos recursos en pro de la mitigación de este impacto.

\section{FUENTES DE FINANCIACIÓN}

Se agradece al docente de la Universidad del Cauca Ronald Alejandro Macuacé Otero por asesorar el presente trabajo de investigación dentro del marco de la asignatura de Economía Ambiental del programa de Ingeniería Ambiental de la Universidad del Cauca.

\section{POTENCIAL CONFLICTO DE INTERESES}

No hay ningún tipo de conflicto de intereses respecto a la publicación de este artículo, el cual es elaborado como requisito para la aprobación de la asignatura de Economía Ambiental del programa de Ingeniería Ambiental de la Universidad del Cauca. 


\section{REFERENCIAS}

- Arbohaín, C. y Garcén, L. Contaminación Visual. Arqchile.cl Articulo En Línea: Link. 2001.

- Bookchin, Murray. Los límites de la ciudad. Ediciones H Blume. Madrid.1974.

- Boff, Leonardo. Daños por contaminación ambiental urbana e inmisiones inmateriales - Una tensión entre viejas y nuevas realidades que el derecho debe plantear y resolver. Argentina. 2013

- Díaz Araujo, María M. Daño por contaminación ambiental urbana. Polución. Impacto Auditivo, Visual y Ambiental, Volumen IV. Página 686. 1997.

- Olivares, Fernando. "Cidadelimpa" y la contaminación publicitaria en la ciudad. ZER: Revista de comunicación del País Vasco. 2009.

- Peña, Mario. La tutela jurídica del paisaje. Revista Judicial número 96, Costa Rica, San José, junio, 2010.

- Pereira, Jackson. La contaminación visual actual de los avisos publicitarios en la ciudad de Bogotá D.C. Universidad Militar de Colombia. 2013.

- Tzonis, Alexander. Hacia un entorno no opresivo. H. Blume Ediciones. Madrid. 1977.

- Universidad del Cauca. Boletín Estadístico. Artículo En Línea: Link . 2013.

1. Ingeniero Ambiental de la Universidad del Cauca. davidalejandro2006@hotmail.com

Para citar este artículo: Mera-Benavides, D.A. (2017). Diagnóstico ambiental de la percepción de la contaminación visual por parte de la población universitaria de la Facultad de Ingeniería Civil y de la Facultad de Ciencias Naturales, Exactas y de la Educación de la Universidad del cauca. Revista Luna Azul, 44, 211-230. DOI: 10.17151/luaz.2017.44.13. de http://200.21.104.25/lunazul/index.php/component/content/article?id=229

Esta obra está bajo una Licencia de Creative Commons Reconocimiento CC BY

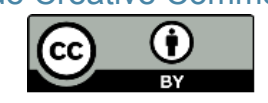

